properly protected. Waterlogging of clothing either from without or within must, as far as possible, be protected, and this means waterproof outer garments, either of a ventilatory fabric or over-trousers and a Kagoule.

If hypothermia becomes established and a person in a party begins to show signs of collapse, treatment should be immediate. It is allowable to move the patient a short distance, if by this either good shelter or a considerable loss of altitude can be achieved: otherwise all cases should be treated where they occur. At the same time as treatment is started help must be sent for. The immediate first aid measure is to prevent further heat loss, and this means placing the patient first in a sleeping bag and then in a thick polythene survival bag. The place chosen should, as far as possible, be shielded from the wind and the head, as well as the rest of the body, should be covered. Fluid, sugar and salt may be given, but alcohol should never be given. Many persons with hypothermia will continue to lose body heat despite these measures and some method of body heating may be required. In the survival situation this is best provided by another body, and a fit member of the party should be put in the bag with the patient. When mountain-rescue assistance arrives the first aid measures are the same as should already have been started and the rescuers will attempt to remove the patient to hospital as rapidly as is consistent with safety. The only additional resuscitative measures which may be employed are the giving of water and salt by intravenous drip and the new method of body heating being examined of heating the air breathed in. If a person suffering from exhaustion/exposure is got to habitation they can be placed in a hot bath, but careful watching is required, as too rapid heating can be dangerous.

If hypothermia is diagnosed as early as possible and the correct first aid measures instituted on the spot, the chances of survival are very good; if, on the other hand, the party tries to press on and get the sufferer to walk to habitation and treatment, the risk of death is high

\section{Conclusions}

The Scottish mountains offer unrivalled facilities for physical exercise, but they must be treated with respect and the sub-arctic nature of the climate recognised.

The complications of mountain exposure should be guarded against by adequate planning before any expedition, knowledge of the capabilities and limitations of the members of the party, and experienced mountain leadership during the expedition.

Diagnosis and treatment of the complications of mountain exposure when these occur should be early and prompt. Any attempt to walk out to help rather than treatment on the spot is likely to result in further complication and maybe death.

\title{
ANNOTATION \\ BLOOD GLUCOSE MEASUREMENTS IN FIELD TRIALS
}

\author{
L. F. Green, B.Sc., A.R.I.C., F.C.S., F.I.F.S.T., Beecham Products, Brentford
}

Blood sampling in the field presents many problems, such as site of sampling, size of sample, how to prepare the blood sample, the effect of storage and the analytical method.

In the light of our experiences, in many different sports situations, the procedure we have adopted to give maximal accuracy may be of interest.

1. Generally, finger incisions by sterile lancet are most satisfactory.

2. $0.1 \mathrm{ml}$ blood is adequate.

3. Zinc uranyl acetate $(0.16 \% \mathrm{w} / \mathrm{v})$, ready prepared by

Boehringer \& Co. (URAC), is the most suitable deproteinizing reagent.

4. Proteinaceous matter should be separated by centrifugation immediately after precipitation.

5. Storage of the deep-frozen supernatant leads to decreasing values and minimum storage is desirable. When comparisons are to be made, the time of storage should be standardised.

6. B-d-glucose, is determined by using an automated glucose oxidase-peroxide method.

By analysis of variance, 27 samples using this procedure, gave a standard deviation of $\pm 1.03 \mathrm{mg} / 100 \mathrm{ml}$ within duplicates. 